Portland State University

PDXScholar

7-14-2014

\title{
Optical Injection Unlocking for Cavity Ringdown Spectroscopy
}

\author{
Gregory A. Bostrom \\ Portland State University, gbostrom@pdx.edu \\ Andrew L. Rice \\ Portland State University, arice@pdx.edu \\ Dean B. Atkinson \\ Portland State University, atkinsond@pdx.edu
}

Follow this and additional works at: https://pdxscholar.library.pdx.edu/phy_fac

Part of the Chemistry Commons, and the Optics Commons Let us know how access to this document benefits you.

Citation Details

Greg Bostrom, Andrew Rice, and Dean Atkinson, "Optical injection unlocking for cavity ringdown spectroscopy," Opt. Lett. 39, 4227-4230 (2014) http://www.opticsinfobase.org/ol/ abstract.cfm?URI=ol-39-14-4227

This Article is brought to you for free and open access. It has been accepted for inclusion in Physics Faculty Publications and Presentations by an authorized administrator of PDXScholar. Please contact us if we can make this document more accessible: pdxscholar@pdx.edu. 


\title{
Optical injection unlocking for cavity ringdown spectroscopy
}

\author{
Greg Bostrom, ${ }^{1, *}$ Andrew Rice, ${ }^{1}$ and Dean Atkinson ${ }^{2}$ \\ ${ }^{1}$ Portland State University, Physics Department, P.O. Box 751, Portland, Oregon 97207-0751, USA \\ ${ }^{2}$ Portland State University, Chemistry Department, P.O. Box 751, Portland, Oregon 97207-0751, USA \\ ${ }^{*}$ Corresponding author: gbostrom@pdx.edu
}

Received May 6, 2014; revised June 13, 2014; accepted June 13, 2014;

posted June 17, 2014 (Doc. ID 211124); published July 14, 2014

\begin{abstract}
Continuous wave cavity ringdown spectroscopy requires a rapid termination of the injection of light into the cavity to initiate the decay (i.e., ringdown) event. We demonstrate a technique that accomplishes this through pulsed optical injection of a second laser into the main laser, resulting in $20-100 \mathrm{MHz}$ frequency shifts in the otherwise cavity-locked main laser sufficient to create ringdown events at $3.5 \mathrm{kHz}$. Data on the frequency shift as a function of both main laser current and relative wavelength are presented, as well as a demonstration that single exponential decays are maintained in the process. (C) 2014 Optical Society of America

OCIS codes: (120.6200) Spectrometers and spectroscopic instrumentation; (140.2020) Diode lasers; (140.3070) Infrared and far-infrared lasers; (140.3520) Lasers, injection-locked; (300.6260) Spectroscopy, diode lasers.

http://dx.doi.org/10.1364/OL.39.004227
\end{abstract}

Cavity ringdown spectroscopy (CRDS) is a sensitive optical extinction technique that uses the exponential decay of light energy from a high-finesse optical cavity to determine the total losses in the cavity. For more details, there are a number of reviews of the technique and its application [1,2]. Energy is built up in the cavity by tuning the laser so that its frequency matches a resonant mode of the cavity. In order to initiate the decay of light out of the cavity, the injection of light into the cavity must be stopped on short time scales (typically $<1 \mu \mathrm{s}$ ). Typically this is accomplished by interrupting the laser through an external optical switch, such as an acousto-optic modulator or electro-optic modulator [3-5]. These devices are costly ( $\$ 2000-\$ 7000)$, especially in the near-IR range. Additionally, the acousto-optic modulator introduces a Doppler frequency shift and can interfere with optical feedback techniques used to lock the laser to the cavity [6]. Electro-optic modulators use electrically controlled polarization switching, which does not shift frequency and therefore is a common choice for pulsing the laser [7]. The requirement for switching hundreds to thousands of volts over times of 10-100 ns complicates the design of these devices. A mechanical method of shifting the cavity off resonance by mounting one of the cavity mirrors on a piezoelectric transducer and applying a voltage was demonstrated in []], but this method could be problematic for systems that employ optical feedback to lock the laser to the cavity since the cavity length is continuously changing. Another technique to modulate the laser intensity is to simply pulse the laser drive current [9], but with this technique the laser driver bandwidth limits the time the laser can be kept off, and the current modulation induces thermal frequency shifts. These issues increase the complexity in the fitting algorithm, the current pulsing timing, and the frequency shifting circuit. Using optoelectronic locking to the cavity, such as the Pound-Drever-Hall method [10], requires additional circuit complexity to turn off the electronic locking during the ringdown and then relock the laser to the cavity $[\underline{8}, \underline{11}, \underline{12}]$.

In this Letter, we present a novel technique to move the ringdown laser off resonance in less than a microsecond, while not interfering with cavity locking, by injecting a second, pulsed diode laser into the main laser. This method does not change the current of the locked laser, allowing it to return to cavity resonance after the disrupting pulse is over. It is also inexpensive and requires only a diode laser, a beam splitter, and a simple pulsing circuit. We first present the method and experimental setup used to demonstrate the technique. Second, we analyze the dependence of the frequency shift on both main laser power and wavelength difference between the pulse laser and main laser. Finally, we demonstrate cavity ringdown using this technique.

A diode laser is susceptible to the introduction of external electromagnetic fields, resulting in a continuum of effects, from chaotic frequency instabilities to frequency stabilization. These effects have been studied fairly extensively [13]. The delayed reinjection of light produced by a laser and spectrally filtered by cavity has been used to lock the diode laser to the cavity for optical feedback cavity-enhanced spectroscopy [14]. The effect of optical injection on a diode laser was included in a review article by Van Tartjwik and Lenstra [15]. The typical application of optical injection is to stabilize and lock a slave laser to a master laser by influencing the refractive index and the electric field inside the slave laser's medium. The change in refractive index directly causes a shift in the lasing frequency (since the frequency is determined by the speed of light in the medium and the cavity length). The locking of the slave laser requires that the master and slave laser be close (typically within a few GHz). However, the refractive index changes (which in turn alter the resonance frequency of the laser cavity) occur over a much wider range, as long as the energy of the photon induces changes in carrier density. The effect of changes in carrier density on the laser materials has been studied by others: see Fig. 3 in [16], Eq. (4) in [17], and [18]. It is this effect that we exploit to shift the frequency in this work.

The experimental setup is shown in Fig. 1. A distributed feedback laser (Mitsubishi, ML725B11F, with an approximate wavelength of $1308 \mathrm{~nm}$ ) to be used for spectroscopy (as the main ringdown laser) is directed into a near-confocal high-finesse optical cavity comprised of 


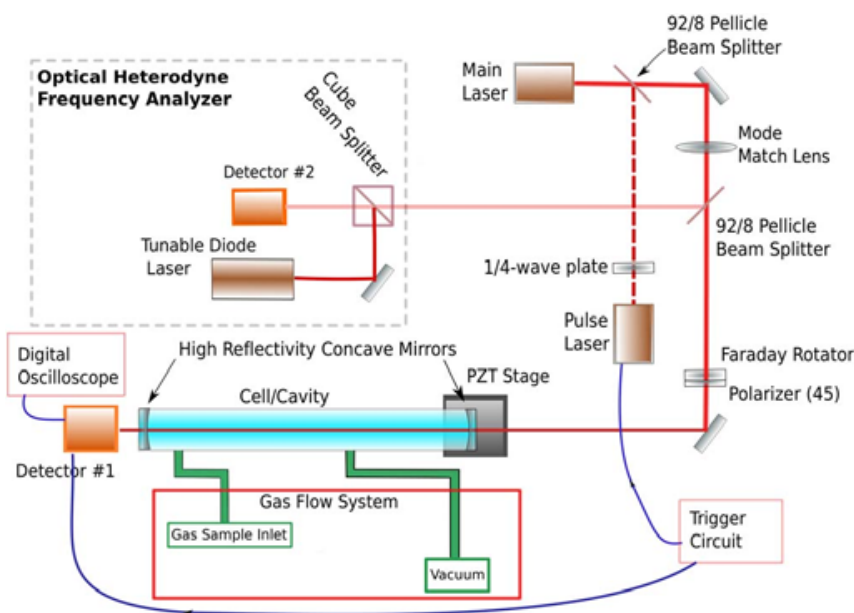

Fig. 1. Diagram of the system. The main laser is injected into the optical cavity for the ringdown measurement. A pulsed laser is aligned to inject light back into the main laser. A simple pulsing/triggering circuit is used to activate the pulsed laser. A tunable diode laser and a secondary detector are used to detect frequency shifts in the main laser using OHD for initial characterization (dashed-boxed region is not a permanent part of the system). A digital oscilloscope measures the cavity output and optical heterodyne signals. The Faraday rotator and polarizer control optical feedback to the main laser to enable locking to the cavity. The quarter-wave plate reduces coherent reflections from the pulsed laser.

two plano-concave mirrors (Newport, \#10CV00SR.60F, reflectivity $>99.97 \%$, yielding a cavity finesse of $>10,000)$. A $92 \% / 8 \%$ beam splitter directs a small fraction of a pulsed Fabry-Perot laser (Mitsubishi, ML725B8F, with an approximate wavelength of $1309 \mathrm{~nm}$ ) into the main laser, while allowing a large fraction of the main laser to pass to the cavity. The Faraday rotator and polarizer in the path are used to control the optical feedback from the cavity to the laser, similar to $[\underline{6}, \underline{9}, \underline{14}]$, enabling the laser to lock to a cavity resonance. Our linear configuration is a simplified version and so is not capable of effectively locking to all cavity modes due to etalon effects of the laser-cavity path. As we are only demonstrating the frequency shifting technique, this configuration is sufficient. A second beam splitter samples the main laser and sends it into an optical heterodyne (OHD) frequency analyzer. This analyzer uses a stable tunable diode laser (New Focus, model 6324, with a linewidth of $300 \mathrm{kHz}$ using a $50 \mathrm{~ms}$ integration time, and a drift of $<5 \mathrm{MHz}$ over $1 \mathrm{~s}$ ) as a reference that mixes with a laser under test on the photodetector (Thorlabs, model PDA255). The mixing produces a beat frequency that indicates the frequency of the laser under test relative to the reference laser. The photodetector signal output is connected to a digital oscilloscope (Tektronix, model TDS-3052) to collect the voltage versus time for frequency analysis using fast Fourier transform (FFT). As long as the beat frequency is within the bandwidth of the photodetector (approximately $100 \mathrm{MHz}$ ), this accurately measures the frequency shift induced by the pulsed laser. The OHD system is only used for experimental characterization and would not be part of an eventual implementation of this technique. When the main laser is unlocked from the cavity, a second photodetector (New Focus, model 1811) at the output of the cavity allows measurement of the exponential decay of the cavity energy (the ringdown) using the digital oscilloscope triggered by the pulsing circuitry.

The pulsed laser is controlled by a simple multivibrator (timing) circuit based on the standard 555 integrated circuit $[19,20]$. The circuit can be configured as either astable (continuously pulses the laser) or monostable (an external transistor-transistor logic (TTL) signal triggers a single laser pulse). For testing and measurement of the frequency shift, we configured the circuit in astable mode. For cavity ringdown measurements, the circuit was configured in monostable mode, triggered by a threshold circuit when the cavity output photodetector exceeded a preset voltage. Two resistors and a capacitor set the nominal $90 \mu$ s pulse width (the length of time the main laser was moved off resonance to observe the ringdown decay). The output voltage from the 555 typically was between 3.5 and $3.75 \mathrm{~V}$, which drove the pulsed laser at an estimated power of $2.5 \mathrm{~mW}$ (barely above the lasing threshold current of $5 \mathrm{~mA}$ ). Alignment and wavelength measurements required higher power, so the laser power was increased to approximately $17 \mathrm{~mW}$ (at a drive current of $35 \mathrm{~mA}$ ). FFT analysis of a typical OHD signal showed the main laser's peak frequency shifted approximately $20 \mathrm{MHz}$ and returned to the original frequency after the pulse. Time domain analysis showed that the frequency shift occurred within 200-500 ns.

Measuring a spectrum would require tuning the frequency of the main ringdown laser, presumably without changing the pulsed laser's output, resulting in changes to the frequency difference between the lasers. To examine the frequency shift of the main laser as a function of the frequency difference between the main and pulsed laser, we tuned the main laser over a $1 \mathrm{~nm}$ range by varying its operating temperature between approximately $15^{\circ} \mathrm{C}$ and $27^{\circ} \mathrm{C}$. The main laser current was fixed at $10.1 \mathrm{~mA}$, about twice the threshold current. Figure 2 shows the results: a variation in frequency shift between 20 and $100 \mathrm{MHz}$ when the pulsed laser is $>0.1 \mathrm{~nm}$ from the main laser. When the pulsed laser is $<0.1 \mathrm{~nm}$ from the main laser, injection-locking effects dominate, rather than changes in refractive index, and the frequency shift was only about $5 \mathrm{MHz}$. Notably the peak frequency shift occurs when the lasers differ by $0.4 \mathrm{~nm}(70 \mathrm{GHz})$. The explanation for this may be related to the peak refractive index changes described in [21] and [투].

For high-resolution spectroscopy, frequency tuning of the main laser is best accomplished by changing the drive current. For the main laser, the frequency change with current was $1.06 \mathrm{GHz} / \mathrm{mA}$. In Fig. 2, we show the main laser frequency shift as a function of drive current. The main laser was initially tuned to $1308.27 \mathrm{~nm}$; the pulsed laser had a measured wavelength of $1309.45 \mathrm{~nm}$. The $32 \mathrm{~mA}$ current range in Fig. 2 represents a frequency range of approximately $34 \mathrm{GHz}$. The average shift induced by the pulsed laser was $30 \mathrm{MHz}$ (range 15$40 \mathrm{MHz}$ ). The change in photon number (and thus refractive index) is proportional to the ratio of injected power to laser power [15], and therefore the smaller frequency shift at higher currents is expected. At lower currents the laser is less stable [13], causing the larger variation in 


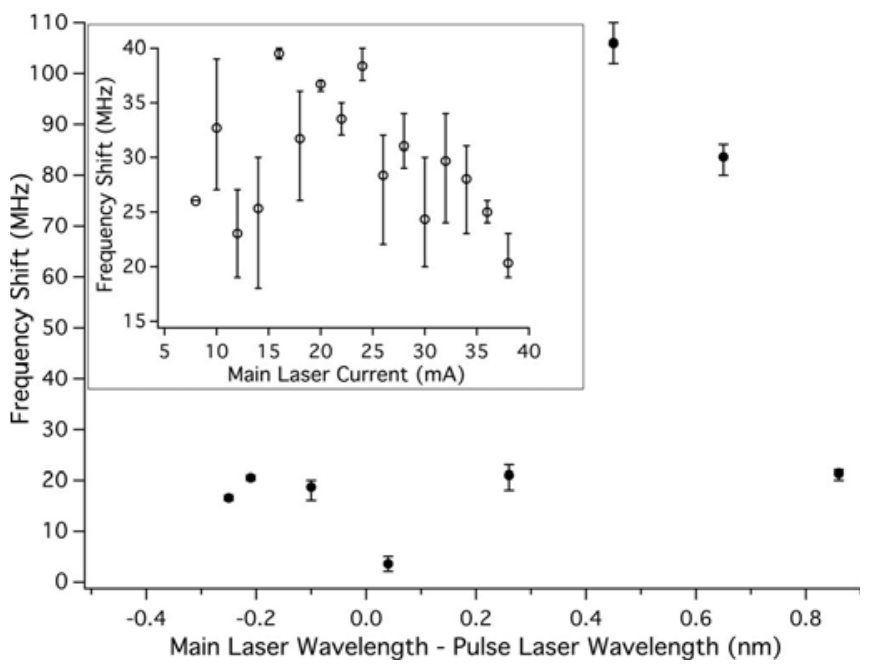

Fig. 2. Induced frequency shift as a function of the wavelength difference between the main laser and the pulse laser. In the main figure, the main laser wavelength is adjusted by changing its temperature. The average of three different measurements taken at each wavelength is shown, with error bars indicating the max and min. Inset: the frequency shift as a function of main laser current, with laser temperature fixed. The range covers approximately $34 \mathrm{GHz}(0.2 \mathrm{~nm})$. Again, average is shown for three different measurements at each current level and error bars indicate max and min.

frequency shifts. For a high-finesse cavity used for cavity ringdown experiments, with a several $\mathrm{kHz}$ linewidth, this is sufficient to shift the laser off resonance without shifting to another cavity mode ( $175 \mathrm{MHz}$ free spectral range for an $85 \mathrm{~cm}$ cavity). The $30 \mathrm{MHz}$ shift for an index of refraction of 3.5 corresponds to a change in refractive index of $5 \times 10^{-7}$. Using the results from [22] the injected power to main laser power ratio is on the order of $10^{-5}$. This is reasonable given optical losses along the pulsed laser path and uncertainty in the spot size of the pulse beam on the main laser facet.

A critical aspect of this approach is that the frequency shift from optical injection of the pulsed laser is sufficient to shift the laser off resonance, unlocking it from the cavity such that an exponential decay is observed. To estimate the extinction ratio (the ratio of the laser power entering the cavity when shifted off resonance to the power when on resonance), we estimated a $<1 \mathrm{MHz}$ linewidth of the main laser using the OHD system. Assuming a Lorentzian shape, this results in a minimum extinction ratio of $30 \mathrm{~dB}$ for a $20 \mathrm{MHz}$ shift and $45 \mathrm{~dB}$ for a $100 \mathrm{MHz}$ shift.

Figure 3(b) shows a typical ringdown triggered after a buildup of energy in the cavity and then induced by the pulsed laser. The single exponential curve fit is also shown. An additional requirement for CRDS is that the laser-cavity interaction be broken with sufficient rapidity (here 200-500 ns) so that a single decay is observed, as demonstrated here by the residuals of the fit (see inset). In subsequent experiments we measured the individual time constants for 17 ringdowns and obtained an average of $12.11 \pm 0.38 \mu \mathrm{s}(1 \sigma)(3.25 \%$ relative standard deviation). Due to the limitations of our current data acquisition system, each ringdown was taken approximately 30 seconds apart, suggesting that the variation
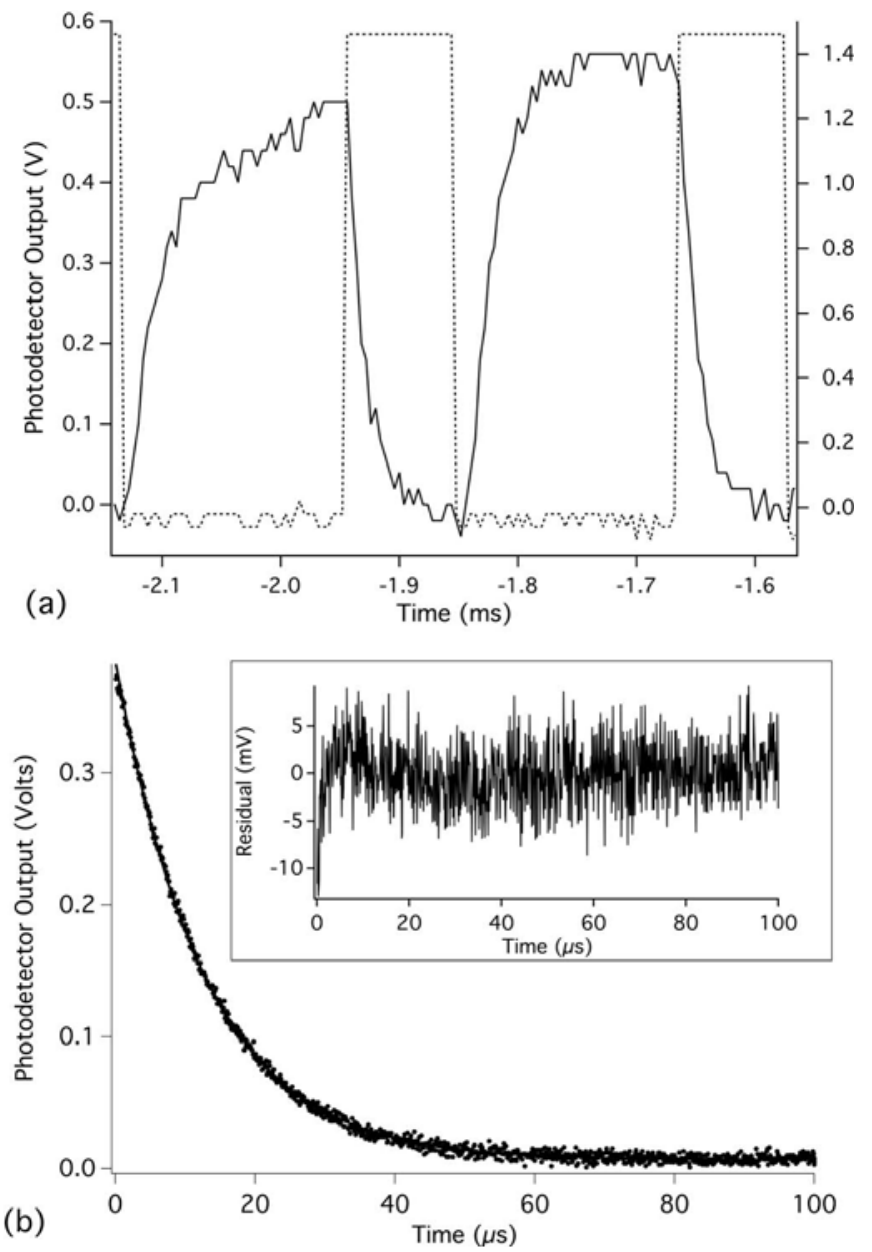

Fig. 3. (a) Two ringdowns taken from the $3.5 \mathrm{kHz}$ rate data described in the text. The solid line shows the output of the cavity, and the dotted gray line shows the pulse laser applied voltage. The cavity builds up energy immediately following the pulse, indicating the main laser has returned to the original frequency and relocked to the cavity. (b) A single ringdown event induced by the pulsed laser frequency shifting the main laser captured on an 8-bit digital oscilloscope (dots). To reduce quantization error, the data was resampled at a rate of $1: 5$. The exponential fit using a Levenberg-Marquardt algorithm is shown (solid line), as well as the residual to the fit (inset).

in time constants is a result of thermal-induced drifting of the cavity length.

Finally, we tested the ability of the technique to repeatedly return the main laser to the resonance frequency of the cavity. We configured the pulsed laser circuit as an astable circuit, producing ringdowns at regular intervals, and achieved ringdown decays at a rate of $3.5 \mathrm{kHz}$. This demonstrates that the pulsing technique does not adversely affect the main laser frequency, which returns to its initial frequency as soon as the pulsed laser shuts off, enabling the laser to relock to the cavity, as shown in Fig. 3(a). Further, applied to CRDS the high data acquisition rates can dramatically improve sampling statistics and precision of measurement (e.g., theoretical improvement of a factor of $\sim 60$ over a $1 \mathrm{~Hz}$ acquisition rate).

In conclusion, we present a technique for frequency shifting a diode laser by up to $100 \mathrm{MHz}$ using a second diode laser that modulates the main laser's refractive index. Our technique, performed over a wide range of laser 
powers and wavelength offsets between the main laser and the pulsing laser, provides a frequency shift sufficient to unlock laser-cavity optical feedback, demonstrating its utility in CRDS. We also demonstrated that the pulsing is effective for inducing a ringdown event for CRDS at $\mathrm{kHz}$ rates.

\section{References}

1. D. B. Atkinson, Analyst 128, 117 (2003).

2. B. A. Paldus and A. A. Kachanov, Can. J. Phys. 83, 975 (2005).

3. D. Romanini, A. A. Kachanov, N. Sadeghi, and F. Stoeckel, Chem. Phys. Lett. 264, 316 (1997).

4. B. Paldus, J. Harris, Jr., J. Martin, J. Xie, and R. Zare, J. Appl. Phys. 82, 3199 (1997).

5. B. L. Fawcett, A. M. Parkes, D. E. Shallcross, and A. J. Orr-Ewing, Phys. Chem. Chem. Phys. 4, 5960 (2002).

6. J. Morville, S. Kassi, M. Chenevier, and D. Romanini, Appl. Phys. B 80, 1027 (2005).

7. N. J. van Leeuwen, J. C. Diettrich, and A. C. Wilson, Appl. Opt. 42, 3670 (2003).

8. J. W. Hahn, Y. S. Yoo, J. Y. Lee, J. W. Kim, and H. W. Lee, Appl. Opt. 38, 1859 (1999).

9. V. Motto-Ros, J. Morville, and P. Rairoux, Appl. Phys. B 87, 531 (2007).

10. R. Drever, J. Hall, F. Kowalski, J. Hough, G. Ford, A. Munley, and H. Ward, Appl. Phys. B 31, 97 (1983).
11. T. G. Spence, M. E. Calzada, H. M. Gardner, E. Leefe, H. B. Fontenot, L. Gilevicius, R. W. Hartsock, T. K. Boyson, and C. C. Harb, Opt. Express 20, 8804 (2012).

12. L. W. H. R. W. Fox, C. W. Oates, and L. Hollberg, in Cavity-Enhanced Spectroscopies, Vol. 40 of Experimental Methods in the Physical Sciences (Academic, 2002).

13. J. Ohtsubo, Semiconductor Lasers: Stability, Instability and Chaos (Springer, 2008).

14. P. Laurent, A. Clairon, and C. Breant, IEEE J. Quantum Electron. 25, 1131 (1989).

15. G. H. M. Van Tartwijk and D. Lenstra, Quantum Semiclass. Opt. 7, 87 (1995).

16. B. R. Bennett, R. A. Soref, and J. A. del Alamo, IEEE J. Quantum Electron. 26, 113 (1990).

17. W. Kowalsky and K. J. Ebeling, Opt. Lett. 12, 1053 (1987).

18. K. Ishida, H. Nakamura, H. Matsumura, T. Kadoi, and H. Inoue, Appl. Phys. Lett. 50, 141 (1987).

19. Texas Instruments, Inc., "LM 555 Datasheet," (2000).

20. M. Gallant, "Pulse circuits for infrared LEDs and visible diode lasers," 2009, http://www.jensign.com/opto/ ledlaserdrivers/.

21. G. H. M. Van Tartwijk, G. Muijres, D. Lenstra, M. P. Van Exter, and J. P. Woerdman, Electron. Lett. 29, 137 (1993).

22. J. Manning, R. Olshansky, and C. B. Su, IEEE J. Quantum Electron. 19, 1525 (1983). 